\title{
Persistence and Progression of Masked Hypertension: A 5-Year Prospective Study
}

\author{
Xavier Trudel, ${ }^{1}$ Alain Milot, ${ }^{1,2}$ and Chantal Brisson ${ }^{1,3}$ \\ ${ }^{1}$ Unité de Recherche en Santé des Populations du Centre de Recherche FRQS du CHU de Québec, CHU de Québec, \\ 1050 Chemin Ste-Foy, Québec, QC, Canada G1S 4L8 \\ ${ }^{2}$ Département de Médecine, Université Laval, Québec, QC, Canada G1V 0A6 \\ ${ }^{3}$ Département de Médecine Sociale et Préventive, Université Laval, Québec, QC, Canada G1V 0A6
}

Correspondence should be addressed to Xavier Trudel; xavier.trudel.1@ulaval.ca

Received 8 August 2013; Revised 13 November 2013; Accepted 14 November 2013

Academic Editor: Tomohiro Katsuya

Copyright ( $) 2013$ Xavier Trudel et al. This is an open access article distributed under the Creative Commons Attribution License, which permits unrestricted use, distribution, and reproduction in any medium, provided the original work is properly cited.

Objectives. To examine masked hypertension persistence over 5 years. Methods. White-collar workers were recruited from three public organizations. Blood pressure (BP) was measured using Spacelabs 90207. Manually operated BP was defined as the mean of the first three readings taken at rest. Ambulatory BP was defined as the mean of the next readings taken every 15 minutes and recorded during working hours. BP was assessed three times over 5 years. Masked hypertension was defined as manually operated BP less than 140 and less than $90 \mathrm{mmHg}$ and ambulatory BP at least 135 or at least $85 \mathrm{mmHg}$. Sustained hypertension was defined as manually operated BP at least 140 or at least $90 \mathrm{mmHg}$ and ambulatory BP at least 135 or at least $85 \mathrm{mmHg}$ or being treated for hypertension. Results. BP measurements were obtained from 1669 participants from whom 232 had masked hypertension at baseline. Persistence of masked hypertension was 38\% and 18.5\%, after 3 and 5 years, respectively. Progression to sustained hypertension was $26 \%$ and $37 \%$, after 3 and 5 years, respectively. Conclusion. Among baseline masked hypertensives, one-third progressed to sustained hypertension and about one out of five remained masked after 5 years, potentially delaying diagnosis and treatment.

\section{Introduction}

The use of ambulatory blood pressure (BP) monitoring has added a new source of information about out-of-office blood pressure. Discrepancies between office and out-of-office blood pressure have resulted in four potential groups of blood pressure status: normotension, sustained hypertension, white coat hypertension, and masked hypertension. According to international guidelines, elevated daytime ambulatory BP (at least 135 or at least $85 \mathrm{mmHg}$ ) in the face of normal office $\mathrm{BP}$ (less than 140 and less than $90 \mathrm{mmHg}$ ) is defined as masked hypertension $(\mathrm{MH})[1,2]$. There has been growing interest in MH over the last decade. Studies have reported that associations between $\mathrm{MH}$ and cardiovascular diseases are as strong as those found for sustained hypertension [3]. Yet, $\mathrm{MH}$ recognition as a clinical entity of its own is still a matter of debate. One main reason for this is the scarce available evidence supporting its persistence over time.
In one prospective study, conducted among patients retrieved from a hospital-based ambulatory BP monitoring database, 11 out of 25 (44\%) initially masked hypertensive patients were still masked when remonitored, while 7 (28\%) had sustained hypertension, after a 1.5-year follow-up [4]. A nested case-control study, which has assessed $\mathrm{MH}$ among youth [5], has reported that 18 out of 34 baseline masked hypertensives became normotensives, 13 had persistent $\mathrm{MH}$ (38\%), and $3(9 \%)$ had sustained hypertension, after a $37-$ month median follow-up. Another study conducted among 50 borderline hypertensives has reported a "fair to moderate" reproducibility of $\mathrm{MH}$, within a 1-week interval $(k=0.47)$ [6]. A fourth study has reported moderate reproducibility of hypertension classification, using morning $(k=0.58)$ and evening $(k=0.46)$ home blood pressure monitoring over 6 months [7]. Finally, two studies conducted among treated hypertensives have reported low $\mathrm{MH}$ persistence over one year and 6 months, respectively $[8,9]$. It is noteworthy that 
treated $\mathrm{MH}$ qualitatively differs from untreated $\mathrm{MH}$. Elevated $\mathrm{ABP}$ in the face of normal clinic BP might be related to poor adherence to treatment or ineffective clinical management explained by the fact that clinic BP is thought to be controlled. Also, to be treated, masked hypertensives must have been identified as having high BP in a clinical setting. Finally, relying on BP levels in treated masked hypertensives to classify them is prone to misclassification, as their blood pressure levels are likely to have been lowered by medication.

Studies about persistence of $\mathrm{MH}$ include a wide range of study groups and vary widely in their definitions, equipment used, measurement procedures, and populations. These studies also share some limitations, such as the small number of included participants [4-6] as well as the short follow-up period $[4,6-8]$. Therefore, it is difficult to determine the real persistence of $\mathrm{MH}$ from the present literature.

The objective of the present study was twofold: (i) to examine, over 5 years, the evolution of MH prevalence in 1669 white-collar workers and (ii) to examine masked hypertension persistence and progression to sustained hypertension, over 5 years, among baseline masked hypertensives.

\section{Method}

2.1. Population and Study Design. The study population was composed of white-collar workers from three public insurance institutions. Workers were recruited for a prospective cohort study aiming to measure the effect of psychosocial environmental factors on the evolution of BP over a 5-year follow-up. Participation rate at baseline was $80.4 \%$. Workers were recruited if they were not pregnant, not suffering from cardiovascular disease, and worked more than $20 \mathrm{~h}$ per week. Data were collected in three steps: at baseline, after 3 years, and after 5 years. At each time, workers completed a selfadministered questionnaire on work characteristics and BP risk factors. Trained staff installed the monitoring device, collected ambulatory BP measurements, and measured weight and height. At baseline, the study sample was composed of 2178 workers. About $23 \%(N=509)$ of the eligible workers at baseline were lost during follow-ups. Final study sample thus involved 1669 participants (711 men and 958 women). This study was approved by the ethical review board of the $\mathrm{CHU}$ de Québec. Participants provided their written informed consent.

2.2. BP Measurements. Blood pressure (BP) was measured using the Spacelabs 90207 oscillometric monitor (Spacelabs Produits Médicaux Ltée, St-Laurent, Quebec, Canada) validated by the independent investigators' protocol, recommended by the Association for the Advancement of Medical Instrumentation and British Hypertension Society $[10,11]$. In a research office at the participant's workplace, the device was installed on the nondominant arm if BP difference measured on both arms was inferior to $10 \mathrm{mmHg}$. Otherwise, it was installed on the arm showing the higher BP level. After the participant had been sitting for 5 minutes, BP was measured three times in the presence of trained personnel using the office check mode of the Spacelabs monitor. Then, BP was measured every $15 \mathrm{~min}$ by the same monitor for the rest of the working day. Manually operated BP (MOBP) was defined as the mean of the first three readings taken at rest and displayed by the Spacelabs monitor. Then, ambulatory BP (ABP) was defined as the mean of the next readings taken every 15 minutes and recorded by the same monitor during regular daytime work.

Participants must have been measured at least 20 times, which is a more stringent restriction than the 14 measurements recommended by international guidelines [12]. Participants were classified into four categories according to the European Society of Hypertension guidelines [2]: normotension, in which MOBP was less than 140 and less than $90 \mathrm{mmHg}$ and ABP was less than 135 and less than $85 \mathrm{mmHg}$; white coat hypertension, in which MOBP was at least 140 or at least $90 \mathrm{mmHg}$ and ABP was less than 135 and less than 85 mmHg; $\mathrm{MH}$, in which MOBP was less than 140 and less than $90 \mathrm{mmHg}$ and ABP was at least 135 or at least $85 \mathrm{mmHg}$; and sustained hypertension, in which MOBP was at least 140 or at least $90 \mathrm{mmHg}$ and $\mathrm{ABP}$ was at least 135 or at least $85 \mathrm{mmHg}$. Participants treated for hypertension were classified as sustained hypertensives. Workers were systematically informed about their hypertensive status between measurements and referred to their physician when their $\mathrm{ABP}$ values were compatible with the diagnosis of hypertension.

2.3. Risk Factors. Lifestyle risk factors examined were cigarette smoking status, BMI, alcohol intake, and sedentary behaviours. Smoking status was defined as the daily consumption of at least one cigarette per day. Body weight and height were measured to calculate BMI $\left(\mathrm{kg} / \mathrm{m}^{2}\right)$. Alcohol intake was measured using the following three categories, related to weekly intake frequency during the past 12 months: less than one drink per week, one to five drinks per week, and six or more drinks per week. Participants were further classified as having sedentary behaviours (yes/no), according to their weekly leisure physical activity frequency ( $<1 /$ week/ $\geq 1$ /week). Age, education, and family history of cardiovascular disease (CVD) were also examined. The definition of the latter group was based on the declaration by the participant of a cardiovascular event, such as angina, myocardial infarction, coronary revascularization, or stroke, suffered by their father, mother, brother, or sister before the age of 60 . The risk factors listed above were evaluated using validated protocols $[13,14]$.

2.4. Analyses. All analyses were conducted using SAS [15]. A tabular analysis was first conducted to describe baseline demographic and lifestyle characteristics for all participants. Comparisons between mean BP (MOBP and ABP) were computed using an analysis of variance. Prevalence estimates for each hypertension category were calculated for the whole study population, at each measurement time. Persistence of $\mathrm{MH}$ and progression to sustained hypertension were examined in two steps: (1) using the within-subject persistence of $\mathrm{MH}$, after 3 years, among baseline masked hypertensives $(N=232)$ and (2) using the within-subject persistence of $\mathrm{MH}$, from 3 to 5 years, among participants with masked hypertension both at baseline and after 3 years $(N=88)$. 


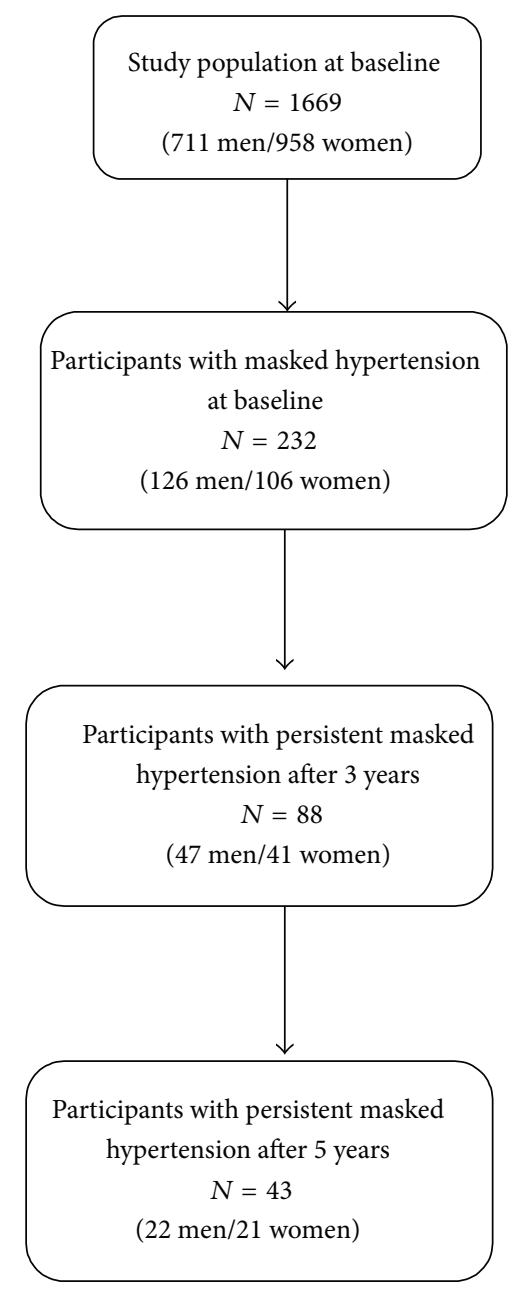

FIGURE 1: Flowchart of masked hypertension within-subject persistence over time.

The overall persistence of $\mathrm{MH}$ after 5 years, among baseline masked hypertensives, was also examined. Figure 1 presents the selection process used to assess within-subject MH persistence. Progression to sustained hypertension was defined as having MOBP at least 140 and at least $90 \mathrm{mmHg}$ and $\mathrm{ABP}$ at least 135 and at least $85 \mathrm{mmHg}$, or being treated for hypertension.

\section{Results}

Table 1 presents the distribution of baseline demographic and lifestyle characteristics for each hypertension category. $\mathrm{MH}$ prevalence was higher among men (17.7\%) compared to women (11.1\%) and increased with age. $\mathrm{MH}$ prevalence was also higher among participants with higher education. Smoking status and sedentary behaviours were not associated with differences among groups $(P>0.05)$, whereas BMI, alcohol intake, and family history of CVD were associated with an increasing proportion of masked hypertension in higher category.

Table 2 presents the mean systolic and diastolic BP, at baseline, by hypertension category and measurement type. In

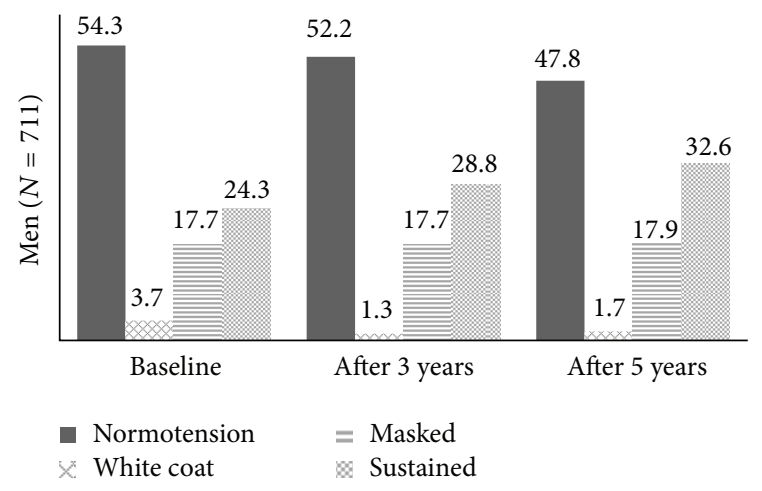

(a)

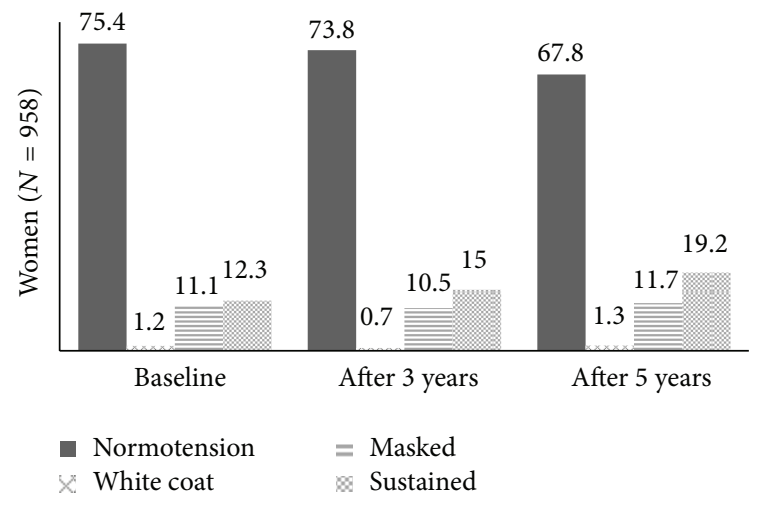

(b)

FIgURE 2: Prevalence of hypertension categories over time for all study participants $(N=1669)$.

men, those with $\mathrm{MH}$ had higher BP levels than normotensive participants for both measurement types, namely, for systolic MOBP, 130.1 versus $123.0 \mathrm{mmHg}$; for diastolic MOBP, 82.5 versus $75.5 \mathrm{mmHg}$; for systolic $\mathrm{ABP}, 134.6$ versus 123.3 $\mathrm{mmHg}$; and for diastolic ABP, 87.8 versus $77.9 \mathrm{mmHg}$. In women, masked hypertensives also had higher BP levels than normotensive, namely, for systolic MOBP, 127.4 versus 116.7 $\mathrm{mmHg}$; for diastolic MOBP, 82.1 versus $73.0 \mathrm{mmHg}$; for systolic ABP, 132.8 versus $118.1 \mathrm{mmHg}$; and for diastolic ABP, 87.4 versus $75.7 \mathrm{mmHg}$.

Figure 2 presents the prevalence of hypertension categories over time, for all study participants $(N=1669)$. Normotension prevalence has decreased among men (from $54.3 \%$ to $47.8 \%$ ) and women (75.4\% to $67.8 \%)$. Conversely, sustained hypertension prevalence has increased among both genders (from $24.3 \%$ to $32.6 \%$ in men and from $12.3 \%$ to $19.2 \%$ in women). $\mathrm{MH}$ prevalence remained stable across all three measurement periods among men (ranging from $17.7 \%$ to $17.9 \%$ ) and women (ranging from 10.5 to $11.7 \%$ ). The proportion of sustained hypertensives who were classified as such because of medication for hypertension rose from $35.3 \%$ to $65.5 \%$ and from $53.4 \%$ to $74.5 \%$ between baseline and last follow-up, in men and women, respectively (not shown).

Figure 3 presents MOBP and ABP means over time, among baseline masked hypertensives $(N=232)$. ABP means were consistently higher than MOBP means across all 
TABLE 1: Description of the study population at baseline.

\begin{tabular}{|c|c|c|c|c|c|}
\hline & Normotension & $\begin{array}{c}\text { White coat } \\
\text { hypertension }\end{array}$ & Masked hypertension & $\begin{array}{c}\text { Sustained } \\
\text { hypertension }\end{array}$ & $P$ \\
\hline Gender & & & & & $<0.001$ \\
\hline Women & $722(75.4)$ & $12(1.3)$ & $106(11.1)$ & $118(12.3)$ & \\
\hline Men & $386(54.3)$ & $26(3.7)$ & $126(17.7)$ & $173(24.3)$ & \\
\hline Age & & & & & $<0.001$ \\
\hline$<40$ & $321(75.9)$ & $13(3.07)$ & $57(13.5)$ & $32(7.6)$ & \\
\hline $40-49$ & $583(68.2)$ & $17(2.0)$ & $115(13.5)$ & $140(16.4)$ & \\
\hline$\geq 50$ & $204(52.2)$ & $8(2.1)$ & $60(15.4)$ & $119(30.4)$ & \\
\hline Education & & & & & 0.0209 \\
\hline Less than college & $287(66.9)$ & $9(2.1)$ & $43(10.0)$ & $90(21.0)$ & \\
\hline College & $340(69.4)$ & $9(1.8)$ & $72(14.7)$ & $69(14.1)$ & \\
\hline University & $481(64.1)$ & $20(2.7)$ & $117(15.6)$ & $132(17.6)$ & \\
\hline Sedentary behaviours & & & & & 0.6673 \\
\hline No & $950(66.3)$ & $35(2.4)$ & $201(14.0)$ & $247(17.3)$ & \\
\hline Yes & $153(66.2)$ & $3(1.3)$ & $31(13.4)$ & $44(19.1)$ & \\
\hline Body mass index $\left(\mathrm{kg} / \mathrm{m}^{2}\right)$ & & & & & $<0.001$ \\
\hline$<25$ & $589(76.1)$ & $11(1.4)$ & $93(12.0)$ & $81(10.5)$ & \\
\hline $25-26.9$ & $196(64.3)$ & $10(3.3)$ & $45(14.8)$ & $54(17.7)$ & \\
\hline$\geq 27$ & $319(54.6)$ & $17(2.9)$ & $93(15.9)$ & $155(26.5)$ & \\
\hline Family history of CVD & & & & & 0.0062 \\
\hline No & $760(69.0)$ & $25(2.3)$ & $146(13.3)$ & $171(15.5)$ & \\
\hline Yes & $314(60.9)$ & $12(2.3)$ & 77 (14.9) & $113(21.9)$ & \\
\hline Alcohol intake & & & & & 0.0003 \\
\hline$<1 /$ week & $369(68.1)$ & $15(2.8)$ & $63(11.6)$ & $95(17.5)$ & \\
\hline 1-5/week & $503(70.5)$ & $13(1.8)$ & $89(12.5)$ & $108(15.2)$ & \\
\hline$\geq 6 /$ week & $236(57.2)$ & $10(2.4)$ & $79(19.1)$ & $88(21.3)$ & \\
\hline Smoking status & & & & & 0.7179 \\
\hline No & $978(66.7)$ & $34(2.3)$ & $204(13.9)$ & $250(17.1)$ & \\
\hline Yes & $130(64.3)$ & $4(2.0)$ & $27(13.4)$ & $41(20.3)$ & \\
\hline
\end{tabular}

Missing values range from 0 to $3.8 \%$. Sociodemographic variables (gender, age, and education) had no missing value.

three measurement times. However, differences between ABP and MOBP decreased from 4.87 to $2.99 \mathrm{mmHg}$ (systolic) and from 5.35 to $4.2 \mathrm{mmHg}$ (diastolic) after 3 years.

Table 3 presents the evolution of hypertension categories, after 3 years, among those 126 men and 106 women who were masked hypertensives at baseline. $\mathrm{MH}$ persistence was $37 \%$ and 39\%, among men and women, respectively. Among these baseline masked hypertensives, 32 men (25\%) and 29 women (27\%) had developed sustained hypertension, after 3 years. When considering hypertension, either masked or sustained, $62 \%$ of men and $66 \%$ of women with $\mathrm{MH}$ at baseline had the condition after 3 years.

Table 4 presents the evolution of hypertension categories, after 5 years. Among participants with masked hypertension at baseline, the overall persistence of masked hypertension was $34.1 \%$ in men and $33 \%$ in women. Progression to sustained hypertension was observed for $31 \%$ and $39.6 \%$ of these baseline masked hypertensives. Among participants with masked hypertension both at baseline and after 3 years, the persistence of $\mathrm{MH}$, from 3 to 5 years, was present in 43 participants out of 88 , who already had the condition for a prolonged period of time (22 men and 21 women). Therefore, the within-subject persistence of masked hypertension after 5 years was $18.5 \%$ (43/232). Progression to sustained hypertension, within 5 years, occurred in $37 \%$ of baseline masked hypertensives (85/232). Among participants with masked hypertension both at baseline and after 3 years, hypertension, either masked or sustained, was present in $72.3 \%$ of men and $80.5 \%$ of women after 5 years.

\section{Discussion}

$\mathrm{MH}$ prevalence remained stable across all three measurement periods, among all study participants. Persistence of $\mathrm{MH}$, however, decreased over time (from $38 \%$ after 3 years to $18.5 \%$ after 5 years). In the present study, persistence rather than reproducibility was chosen to describe the evolution of $\mathrm{MH}$, 
TABLE 2: Clinical characteristics at baseline according to hypertension category.

\begin{tabular}{|c|c|c|c|c|}
\hline & Normotension & White coat hypertension & Masked hypertension & Sustained hypertension \\
\hline $\operatorname{Men}(N=711)$ & $N=386$ & $N=26$ & $N=126$ & $N=173$ \\
\hline \multicolumn{5}{|c|}{ Manually operated blood pressure $(\mathrm{mmHg})$} \\
\hline Systolic & $123.0 \pm 8$ & $141.8 \pm 5$ & $130.1 \pm 6$ & $142.9 \pm 12$ \\
\hline Diastolic & $75.5 \pm 6$ & $86.2 \pm 7$ & $82.5 \pm 5$ & $89.5 \pm 9$ \\
\hline Pulse pressure & $47.5 \pm 7$ & $55.7 \pm 8$ & $47.7 \pm 6$ & $53.4 \pm 9$ \\
\hline \multicolumn{5}{|c|}{ Ambulatory blood pressure (mmHg) } \\
\hline Systolic & $123.3 \pm 6$ & $129.4 \pm 4$ & $134.6 \pm 6$ & $139.8 \pm 11$ \\
\hline Diastolic & $77.9 \pm 4$ & $80.0 \pm 3$ & $87.8 \pm 4$ & $89.6 \pm 8$ \\
\hline Pulse pressure & $45.4 \pm 5$ & $49.4 \pm 4$ & $46.8 \pm 6$ & $50.2 \pm 7$ \\
\hline Women $(N=958)$ & $N=722$ & $N=12$ & $N=106$ & $N=118$ \\
\hline \multicolumn{5}{|c|}{ Manually operated blood pressure $(\mathrm{mmHg})$} \\
\hline Systolic & $116.7 \pm 9$ & $138.9 \pm 8$ & $127.4 \pm 7$ & $143.4 \pm 14$ \\
\hline Diastolic & $73.0 \pm 7$ & $88.7 \pm 5$ & $82.1 \pm 5$ & $92.8 \pm 10$ \\
\hline Pulse pressure & $43.7 \pm 6$ & $50.2 \pm 12$ & $45.3 \pm 7$ & $50.6 \pm 12$ \\
\hline \multicolumn{5}{|c|}{ Ambulatory blood pressure ( $\mathrm{mmHg}$ ) } \\
\hline Systolic & $118.1 \pm 7$ & $126.5 \pm 6$ & $132.8 \pm 7$ & $140.6 \pm 13$ \\
\hline Diastolic & $75.7 \pm 5$ & $80.1 \pm 3$ & $87.4 \pm 4$ & $91.5 \pm 10$ \\
\hline Pulse pressure & $42.3 \pm 5$ & $46.4 \pm 6$ & $45.3 \pm 7$ & $49.1 \pm 9$ \\
\hline
\end{tabular}

One hundred and twenty-three (53\%) of masked hypertensives were diagnosed from diastolic BP only. Twenty eight (12\%) of masked hypertensives were diagnosed from systolic BP only. Eighty-one (35\%) of masked hypertensives were diagnosed from both BP types.

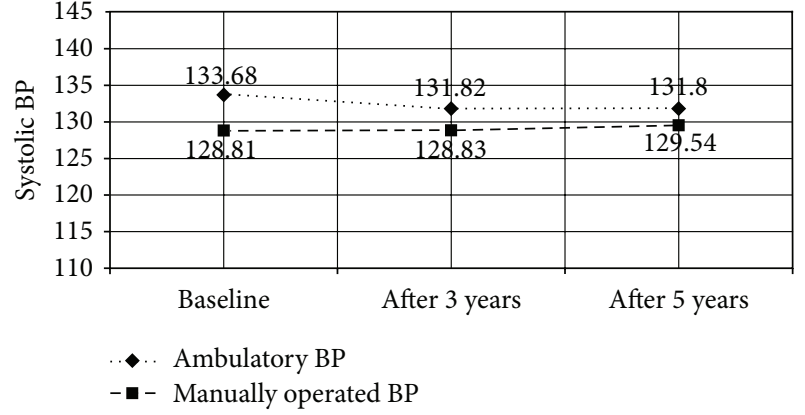

(a)

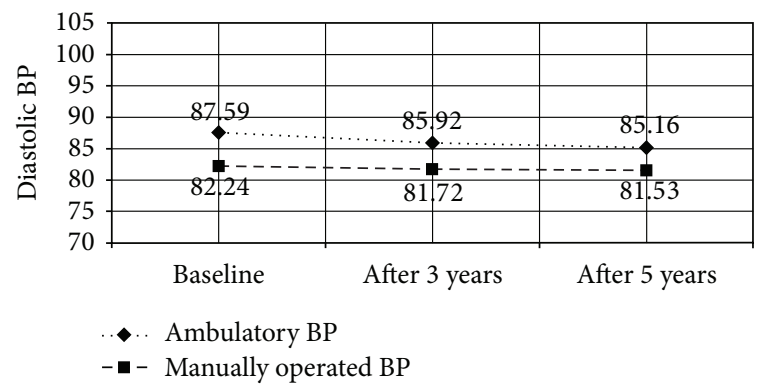

(b)

FIGURE 3: Baseline masked hypertensives ambulatory and manually operated blood pressure means over time $(N=232)$.

as persistence more closely refers to the natural history of the condition. In this perspective, participant with $\mathrm{MH}$ at baseline could be free of this condition after 3 years, not because $\mathrm{MH}$ is not reproducible but because changes occurred in their characteristics, environment, or clinical management.

In the literature, $\mathrm{MH}$ has been hypothesized to be a transient status which progresses to sustained (and detectable) hypertension when a sufficient time lapse has occurred [16]. The present study provides some evidence supporting this hypothesis, as about $37 \%$ of baseline masked hypertensives progressed to sustained hypertension over time. The study however also demonstrates that a significant proportion of baseline masked hypertensives remained as such after 5 years. It argues in favor of $\mathrm{MH}$ being an important clinical issue: about one-third of masked hypertensives progressed to sustained hypertension, and about one out of five remained masked after 5 years. Finally, the results of the present study also suggest that persistent masked hypertensives might be more likely to have the condition over a prolonged period of time, as one-half of participants with masked hypertension both at baseline and after 3 years still had $\mathrm{MH}$ after 5 years.

Among baseline masked hypertensives, mean $\mathrm{ABP}$ values were lower by the years, while MOBP was stable. Two reasons might explain why mean $\mathrm{ABP}$ values were lower. Firstly, about $1 / 3$ of these baseline $\mathrm{MH}$ regressed to normotension from baseline to 3 years. Secondly, a proportion of those baseline masked hypertensives who progressed to sustained hypertension were treated, lowering their BP values. The stability of MOBP could be attributable to a lower precision caused by the limited number of measurements, compared to ABP.

Our study has a number of strengths including its high participation rate at baseline, its high retention rate over 5 years, and its sizeable sample size, compared to previous studies on this topic [17], for both genders. Also, in our study, 
TABLE 3: Hypertension categories evolution from baseline to 3 years among participants with masked hypertension at baseline $(N=232)$.

\begin{tabular}{lccccc}
\hline & $N$ & $\begin{array}{c}\text { Regression to } \\
\text { normotension }\end{array}$ & $\begin{array}{c}\text { Transition to white coat } \\
\text { hypertension }\end{array}$ & $\begin{array}{c}\text { Persistence of masked } \\
\text { hypertension }\end{array}$ & $\begin{array}{c}\text { Progression to sustained } \\
\text { hypertension }\end{array}$ \\
\hline Men & 126 & $47(37.3 \%)$ & 0 & $47(37.3 \%)$ & $32(25.4 \%)$ \\
Women & 106 & $35(33 \%)$ & $1(0.9 \%)$ & $41(38.7 \%)$ & $29(27.4 \%)$ \\
\hline Total & 232 & $82(35.3 \%)$ & $1(0.5 \%)$ & $88(37.9 \%)$ & $61(26.3 \%)$ \\
\hline
\end{tabular}

TABLE 4: Hypertension categories evolution after 5 years.

\begin{tabular}{lccccc}
\hline & $N$ & $\begin{array}{c}\text { Regression to } \\
\text { normotension }\end{array}$ & $\begin{array}{c}\text { Transition to white coat } \\
\text { hypertension }\end{array}$ & $\begin{array}{c}\text { Persistence of masked } \\
\text { hypertension }\end{array}$ & $\begin{array}{c}\text { Progression to sustained } \\
\text { hypertension }\end{array}$ \\
\hline \multirow{2}{*}{ Men } & 126 & $43(34.1 \%)$ & From baseline to 5 years $(N=232)$ & \\
Women & 106 & $29(27.4 \%)$ & $1(0.8 \%)$ & $43(34.1 \%)$ & $39(31 \%)$ \\
\hline Total & 232 & $72(31.0 \%)$ & 0 & $78(33.6 \%)$ & $42(39.6 \%)$ \\
\hline \multirow{2}{*}{ Men } & 47 & $13(27.7 \%)$ & $1(0.5 \%)$ & $22(46.8 \%)$ & $12(25.5 \%)$ \\
Women & 41 & $8(19.5 \%)$ & Within-subject masked hypertension persistence $(N=88)^{*}$ & $12(29.3 \%)$ \\
\hline Total & 88 & $21(23.9 \%)$ & 0 & $21(51.2 \%)$ & $24(27.3 \%)$ \\
\hline
\end{tabular}

${ }^{*}$ Hypertension categories evolution from 3 to 5 years among participants with masked hypertension both at baseline and after 3 years.

both MOBP and ABP were assessed using the same oscillometric device in the same environmental settings (i.e., the workplace). Methods used to identify $\mathrm{MH}$ in previous studies generally imply different devices, which could have influenced prevalence. Finally, the present study examined $\mathrm{MH}$ persistence in a sizeable cohort of workers over 5 years, using a 3-time measurement design. To our knowledge, this has never been done previously.

Our study also has limitations. First, as stated earlier, participants were systematically informed about their hypertensive status between measurements and referred to their physician when their ABP values were compatible with the diagnosis of hypertension. This screening effect might have led to underestimation of $\mathrm{MH}$ persistence to the profit of sustained hypertension given that a proportion of baseline masked hypertensives could have sought medical care for their otherwise unknown condition and therefore be classified as sustained hypertensive at follow-ups. In the present study, MH persistence estimate was nonetheless within the range of previous reports $[4,5]$. It should however be noted that comparability of these estimates across studies is not straightforward, given the heterogeneous populations, the variety of procedure used to measure $\mathrm{BP}$, and the different lengths of follow-up. The persistence of MH could also have been underestimated due to a selection bias in loss at followup. Although no significant difference was found between losses and participants regarding hypertension classification at baseline, losses at follow-up had higher systolic and diastolic MOBP at baseline $(126.4 / 79.0 \mathrm{mmHg}$ versus $124.6 / 77.8 \mathrm{mmHg}, P<0.05)$, as well as higher systolic ABP (126.2 versus $125.0 \mathrm{mmHg}, P<0.05$ ). They also had a higher body mass index (mean BMI $=27.0$ versus $25.0 \mathrm{~kg} / \mathrm{m}^{2}$ ).
This might suggest that workers who were lost had a worse cardiovascular profile than study participants which could have underestimated the persistence of $\mathrm{MH}$. However, the retention rate was high $(77 \%)$, limiting the possibility of an important underestimation of $\mathrm{MH}$ persistence. Finally, the external validity of this study is potentially limited due to the fact that the population was entirely composed of white-collar workers. Further research is needed to investigate the extent to which our findings are generalized to other employees and other populations.

It is also of interest to note that white coat hypertension prevalence was very low. As mentioned in a previous article [18], the low prevalence of white coat hypertension may partially be attributable to the method used to collect MOBP measurements and characteristics of the population being studied. Indeed, MOBP measurements were made by a trained technician, instead of a physician, and at work, rather than in a medical environment. Also, white coat hypertension is more often detected in older people, and one of the predictors of a positive white coat effect has been previously given a diagnosis of hypertension [19].

In the present study, the prevalence of $\mathrm{MH}$ at baseline, $11 \%$ in women and $18 \%$ in men, was within the range of previous reports $[17,20]$. This prevalence, applied to the adult middleaged population, might amount to more than 20 million people in the case of the United States [1]. Considering that one masked hypertensive out of three might evolve toward sustained hypertension and one out of five may remain masked after 5 years, this could translate in more than 10 million people for whom diagnosis and treatment would be delayed. This estimate is most likely underestimated considering that participants were referred to their physician when their ABP 
values, at baseline and at 3 years, were compatible with the diagnosis of hypertension. As for persistent masked hypertensives, many questions remain to be answered regarding their cardiovascular risk profile. More studies are needed to clarify this issue and to determine whether improvement in $\mathrm{MH}$ screening could lead to beneficial effect on CVD morbidity and mortality.

\section{Conclusion}

Among baseline masked hypertensives, hypertension, either masked or sustained, was present in more than half after 5 years, as one-third progressed to sustained hypertension and about one out of five remained masked. These individuals might be at particular risk for cardiovascular disease because of delays in diagnosis and treatment.

\section{Conflict of Interests}

All authors declare that they have no conflict of interests.

\section{Acknowledgments}

This research was supported by a grant from the Canadian Institutes of Health Research. Dr. Brisson held a Canadian Institutes of Health Research Scientist award when this work was conducted. Part of the work from this paper has been previously presented in a poster session (2013, January): Persistence and Progression of Masked Hypertension. Poster session presented at the 21th Scientific Meeting of the Sociéte Québécoise d'Hypertension Artérielle (SQHA), Montréal, Canada. Part of the work from this paper has also been presented in an oral session (2012, October): Reproducibility of Masked Hypertension. Oral session presented at the 2012 Canadian Hypertension Congress, Toronto, Canada.

\section{References}

[1] T. G. Pickering, K. Davidson, W. Gerin, and J. E. Schwartz, "Masked hypertension," Hypertension, vol. 40, no. 6, pp. 795796, 2002.

[2] E. O’Brien, R. Asmar, L. Beilin et al., "Practice guidelines of the European Society of Hypertension for clinic, ambulatory and self blood pressure measurement," Journal of Hypertension, vol. 23, no. 4, pp. 697-701, 2005.

[3] R. H. Fagard and V. A. Cornelissen, "Incidence of cardiovascular events in white-coat, masked and sustained hypertension versus true normotension: a meta-analysis," Journal of Hypertension, vol. 25, no. 11, pp. 2193-2198, 2007.

[4] I. Z. Ben-Dov, L. Ben-Arie, J. Mekler, and M. Bursztyn, "Reproducibility of white-coat and masked hypertension in ambulatory BP monitoring," International Journal of Cardiology, vol. 117, no. 3, pp. 355-359, 2007.

[5] E. Lurbe, I. Torro, V. Alvarez et al., "Prevalence, persistence, and clinical significance of masked hypertension in youth," Hypertension, vol. 45, no. 4, pp. 493-498, 2005.

[6] A. J. Viera, A. L. Hinderliter, A. V. Kshirsagar, J. Fine, and R. Dominik, "Reproducibility of masked hypertension in adults with untreated borderline office blood pressure: comparison of ambulatory and home monitoring," American Journal of Hypertension, vol. 23, no. 11, pp. 1190-1197, 2010.

[7] H. Kawabe and I. Saito, "Reproducibility of masked hypertension determined from morning and evening home blood pressure measurements over a 6-month period," Hypertension Research, vol. 30, no. 9, pp. 845-851, 2007.

[8] W. J. Verberk, T. Thien, A. A. Kroon et al., "Prevalence and persistence of masked hypertension in treated hypertensive patients," American Journal of Hypertension, vol. 20, no. 12, pp. 1258-1265, 2007.

[9] M. G. Myers, M. Godwin, M. Dawes et al., "The conventional versus automated measurement of blood pressure in the office (CAMBO) trial: masked hypertension sub-study," Journal of Hypertension, vol. 30, pp. 1937-1941, 2012.

[10] E. O'Brien, F. Mee, P. Owens et al., "Use and interpretation of ambulatory blood pressure monitoring: recommendations of the British Hypertension Society," British Medical Journal, vol. 320, no. 7242, pp. 1128-1134, 2000.

[11] E. O'Brien, F. Mee, N. Atkins, and K. O’Malley, “Accuracy of the SpaceLabs 90207 determined by the British Hypertension Society protocol," Journal of Hypertension, vol. 9, no. 5, pp. S25-S31, 1991.

[12] E. O’Brien, R. Asmar, L. Beilin et al., "European Society of Hypertension recommendations for conventional, ambulatory and home blood pressure measurement," Journal of Hypertension, vol. 21, no. 5, pp. 821-848, 2003.

[13] C. Daveluy, L. Pica, N. Audet, R. Courtemanche, and F. Lapointe, Enquête Sociale Et de Santé 1998, Institut de la statistique du Québec, Québec, Canada, 2nd edition, 2000.

[14] S. Québec, "Enquête québécoise sur la santé cardiovasculaire 1990," Rapport Final, 1993.

[15] S. A. S. Institute Inc, The SAS System for Sun OS, Version 9.1.3, SAS Institute Inc, Cary, NC, USA, 2007.

[16] G. Mancia, M. Bombelli, R. Facchetti et al., "Long-term risk of sustained hypertension in white-coat or masked hypertension," Hypertension, vol. 54, no. 2, pp. 226-232, 2009.

[17] W. J. Verberk, T. Thien, and P. W. de Leeuw, "Masked hypertension, a review of the literature," Blood Pressure Monitoring, vol. 12, no. 4, pp. 267-273, 2007.

[18] X. Trudel, C. Brisson, B. Larocque, and A. Milot, "Masked hypertension: different blood pressure measurement methodology and risk factors in a working population," Journal of Hypertension, vol. 27, no. 8, pp. 1560-1567, 2009.

[19] T. G. Pickering, W. Gerin, J. E. Schwartz, T. M. Spruill, and K. W. Davidson, "Franz Volhard lecture: should doctors still measure blood pressure? the missing patients with masked hypertension," Journal of Hypertension, vol. 26, no. 12, pp. 2259-2267, 2008.

[20] T. G. Pickering, K. Eguchi, and K. Kario, "Masked hypertension: a review," Hypertension Research, vol. 30, no. 6, pp. 479-488, 2007. 


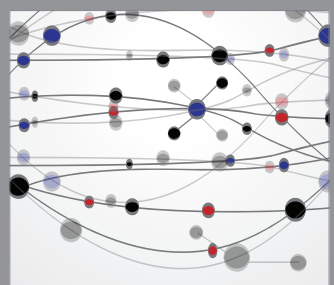

The Scientific World Journal
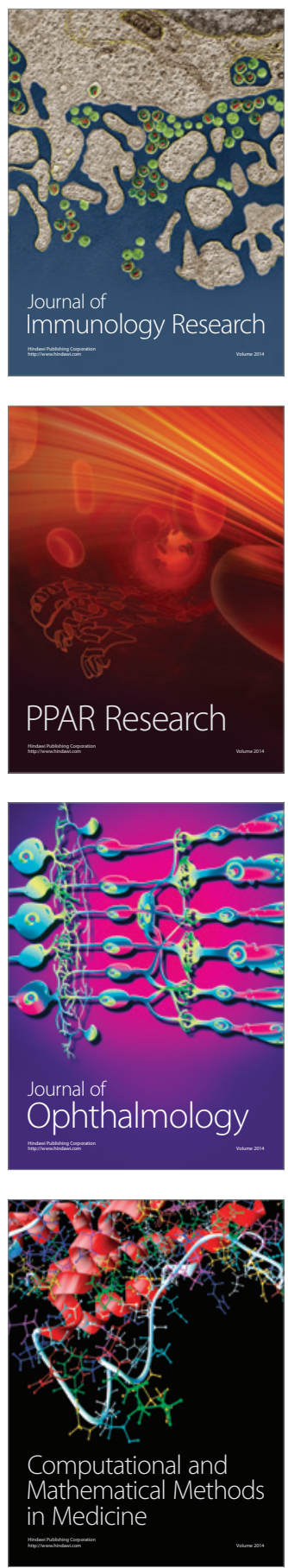

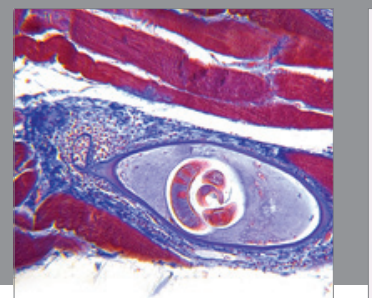

Gastroenterology

Research and Practice
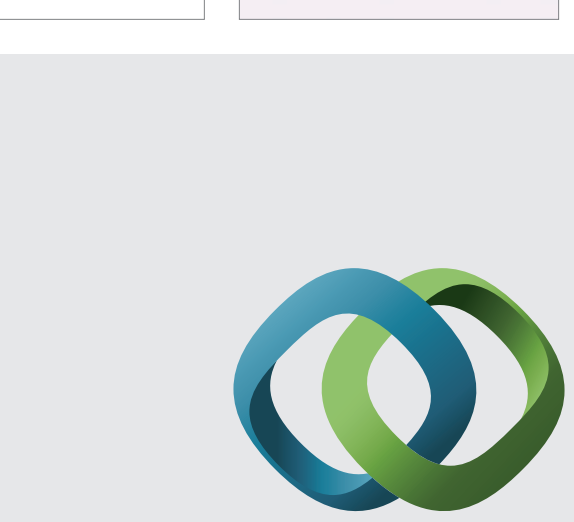

\section{Hindawi}

Submit your manuscripts at

http://www.hindawi.com
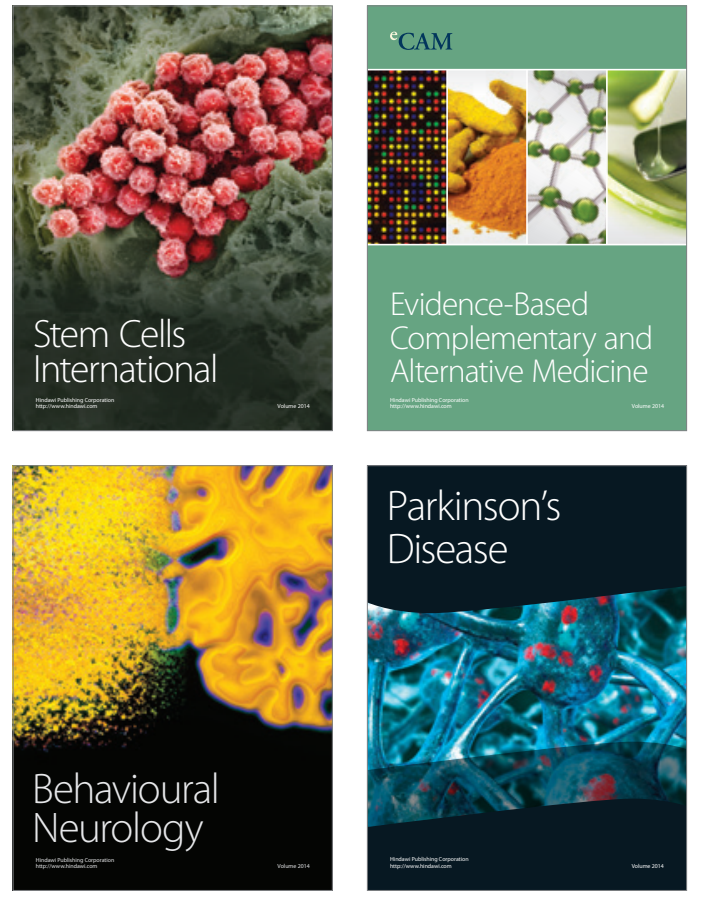
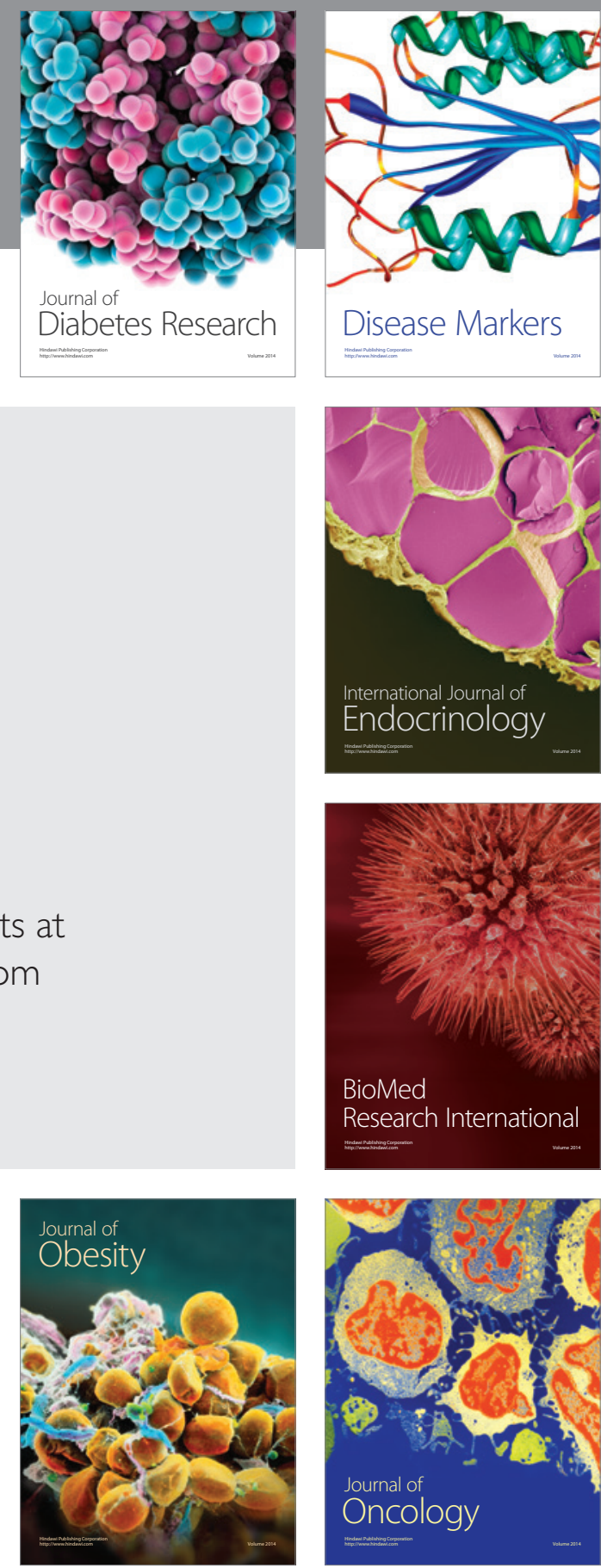

Disease Markers
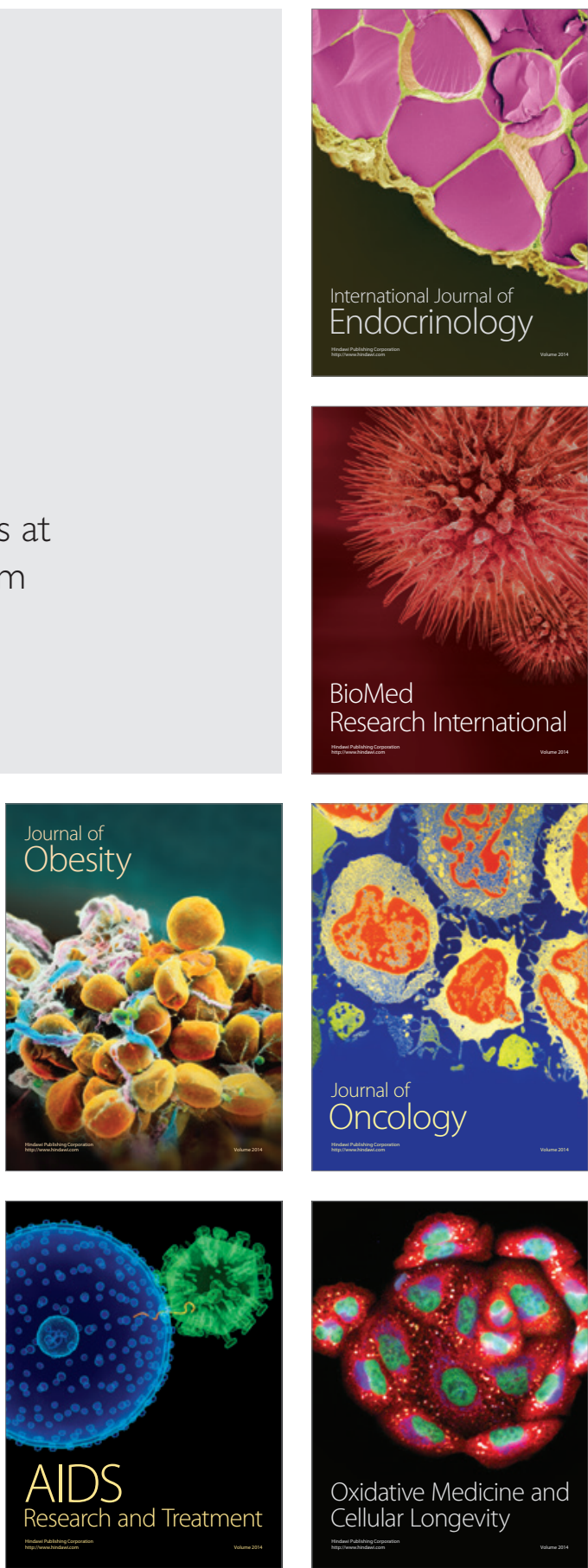